\title{
Porphyromonas gingivalis Resistance to Polymyxin B Is Deter- mined by the Lipid A 4'-Phosphatase, PGN_0524
}

\author{
Stephen R. Coats*, Thao T. To, Sumita Jain, Pamela H. Braham, Richard P. Darveau
}

Department of Periodontics, School of Dentistry, University of Washington, Seattle, USA

\begin{abstract}
Stephen R. Coats, Thao T. To, Sumita Jain, Pamela H. Braham, Richard P. Darveau. Porphyromonas gingivalis Resistance to Polymyxin B Is Determined by the Lipid A 4'-Phosphatase, PGN_0524. International Journal of Oral Science, 1(3): 126-135, 2009
\end{abstract}

Aim To elucidate the genetic basis for the pronounced resistance that the oral pathogen, Porphyromonas gingivalis ( $P$. gingivalis), exhibits towards the cationic antimicrobial peptide, polymyxin B.

Methodology A genetic screen of $P$. gingivalis clones generated by a Tn4400'-based random insertion mutagenesis strategy was performed to identify bacteria harboring novel genetic mutations that render $P$. gingivalis susceptible to killing by the cationic antimicrobial peptide, polymyxin $\mathrm{B}$ (PMB, $50 \mu \mathrm{g} \cdot \mathrm{mL}^{-1}$ ).

Results $P$. gingivalis (ATCC 33277) is unusually resistant to the cationic antimicrobial peptide, $\mathrm{PMB}$ at relatively high concentrations $\left(200 \mu \mathrm{g} \cdot \mathrm{mL}^{-1}\right)$. Approximately 2,700 independent Tn4400'-derived mutants of $P$. gingivalis were examined for increased sensitivity to PMB killing at a relatively low dose $\left(50 \mu \mathrm{g} \cdot \mathrm{mL}^{-1}\right)$. A single PMB-sensitive mutant was obtained in this phenotypic screen. We determined that the Tn4400' transposon was integrated into the gene encoding the lipid A 4'-phosphatase, PGN_0524, demonstrating that this insertion event was responsible for its increased susceptibility of this clone to PMB-dependent killing. The resulting mutant strain, designated 0524-Tn4400', was highly sensitive to PMB killing relative to wild-type $P$. gingivalis, and exhibited the same sensitivity as the previously characterized strain, $0524 \mathrm{KO}$, which bears a genetically engineered deletion in the PGN_0524 locus. Positive ion mass spectrometric structural (MALDI-TOF MS) analyses revealed that lipid A isolates from 0524-Tn4400' and 0524KO strains displayed strikingly similar MALDI-TOF MS spectra that were substantially different from the wildtype $P$. gingivalis lipid A spectrum. Finally, intact 0524Tn4400' and 0524KO mutant bacteria, as well as their corresponding LPS isolates, were significantly more potent in stimulating Toll-like receptor 4 (TLR4)-dependent E-selectin expression in human endothelial cells relative to intact wild-type $P$. gingivalis or its corresponding LPS isolate.

Conclusion The combined molecular evidence provided in this report suggests that PGN_0524, a lipid A 4'-phosphatase, is the sole genetic element conferring the ability of the periodontopathogen, $P$. gingivalis, to evade the killing activity of cationic antimicrobial peptides, such as PMB. These data strongly implicate PGN_0524 as a critical virulence factor for the ability of $P$. gingivalis to evade front-line host innate defenses that are dependent upon cationic antimicrobial peptide activity and TLR4 sensing.

Keywords $P$. gingivalis, antimicrobial peptide, lipid A phosphatase, polymyxin $\mathrm{B}$, transposon, lipopolysaccharide
Document code: A

CLC number: R378.99

\section{Introduction}

Porphyromonas gingivalis ( $P$. gingivalis), a prominent Gram-negative oral bacterium in the human oral cavity, is believed to play a fundamental role in the development and progression of periodontal
Received Aug. 2, 2009; Revision accepted Aug. 30, 2009

disease in the host based upon a substantial amount of clinical evidence (Socransky et al., 1994; Socransky et al., 1998). Multiple potential virulence factors have been identified in $P$. gingivalis that may contribute to its pathogenicity. These factors include fimbriae, which are utilized for host cell 
invasion and subversion of Toll-like receptor responses (Njoroge et al., 1997; Hajishengallis et $a l ., 2008)$ and cysteine proteases which destroy both host extra-cellular matrix components and host innate immune mediators (Takii et al., 2005). Notably, the lipopolysaccharide (LPS) of $P$. gingivalis exhibits an unusually low endotoxic potency in its ability to stimulate host innate immune defenses relative to the potencies that are associated with classic responses elicited by LPS derived from Gram-negative bacteria such as Escherichia coli (E. coli) (Mansheim et al., 1978; Nair et al., 1983). A number of independent studies have implicated this unusual bacterial LPS as potentially playing a role in the ability of $P$. gingivalis to either evade or subvert host innate immune responses. For example, early studies conducted in our laboratory indicated that not only did $P$. gingivalis LPS fail to elicit strong host innate immune responses such as E-selectin induction, but it also antagonized the immunostimulatory activity of agonsitic LPS (Darveau et al., 1995). Subsequent investigations demonstrated that the antagonistic capacity displayed by $P$. gingivalis LPS isolates was due to competitive inhibition of agonistic LPS interaction at the host Toll-like receptor 4 (TLR4) complex (Hajishengallis et al., 2002; Yoshimura et al., 2002; Coats et al., 2003; Coats et al., 2005; Coats et al., 2007), which forms a major host sensing mechanism to Gram-negative bacterial infections (Beutler, 2000; Munford et al., 2006).

Gram-negative bacteria typically produce a mature lipid A bearing di-phosphorylated lipid A structures capable of activating TLR 4 (Poltorak et al., 1998; Raetz et al., 2007). Structural modifications of lipid A including dephosphorylation and deacylation (Peterson et al., 1987; Kovach et al., 1990; Rietschel et al., 1994) can markedly reduce the ability of the LPS to stimulate pro-inflammatory host TLR4 responses (Kawasaki et al., 2004; MataHaro et al., 2007), produce lipid A antagonists (Kovach et al., 1990), or enhance the ability of the bacterium to resist cationic antimicrobial peptides such as polymyxin B (PMB) presumably through the loss of negative charge from either the lipid A 1- or 4'-phosphate (Tran et al., 2006; Wang et al., 2007). Following more than a decade of intensive inquiry aimed at understanding the molecular basis for the low reactivity associated with $P$. gingivalis
LPS, it is now apparent that the distinctive low endotoxic potential associated with the LPS of this bacterium is due to post-synthetic modifications of the lipid A component of its mature LPS including lipid A 1- and 4'-dephosphorylation and lipid A 3'-O-deacylation (Coats et al., 2009). It has previously been shown that $P$. gingivalis synthesizes LPS containing a diphosphorylated, penta-acylated lipid A structure that is capable of promoting a relatively robust innate immune response (Kumada et al., 1995; Kumada et al., 2008). The immunostimulatory potential of the di-phosphorylated, penta-acylated lipid A is greatly reduced by the activity of endogenous lipid A 1- and 4'-phosphatases combined with an unidentified 3'-O-deacylase activity to generate a mono-phosphorylated, tetraacylated lipid A TLR4 antagonist and a novel non-phosphorylated, tetra-acylated lipid A that is highly inert in its ability to stimulate TLR4 responses in human endothelial cells or innate immune responses in monocytes (Rangarajan et al., 2008; Coats et al., 2009). The lipid A 1-phosphatase activity in $P$. gingivalis ATCC 33277, encoded by PGN_1713 (Naito et al., 2008; Coats et al., 2009) (previously denoted PG1773 based upon the W83 genomic sequence), is involved in the hemin-dependent production of the lipid A antagonist which is capable of inhibiting agonistic LPS isolates from both $P$. gingivalis and E. coli. The lipid A 4'-phosphatase activity, encoded by PGN_0524 (Naito et al., 2008; Coats et al., 2009) (previously denoted PG1587 based upon the W83 genomic sequence), appears to be required for subsequent 3'-O-deacylation of the penta-acylated lipid A precursor by an unidentified deacylase activity (Coats et al., 2009).

Experiments performed with $P$. gingivalis bearing a deletion of PGN_0524, 0524KO (previously denoted $1587 \mathrm{KO}$ ), revealed that this lipid A 4'-phosphatase activity suppresses the ability of $P$. gingivalis LPS to activate TLR4 by promoting the formation of non-phosphorylated and mono-phosphorylated tetra-acylated lipid A from a pentaacylated lipid A precursor. Another prominent feature of the $0524 \mathrm{KO}$ strain is that it is highly sensitive to the killing action of the cationic antimicrobial peptide, PMB as compared to the isogenic wild-type strain (Coats et al., 2009). This finding suggests that lipid A 4'-phosphatase 
modification of the lipid A structure reduces the ability of $P$. gingivalis to stimulate TLR4 and establishes its resistance to cationic antimicrobial peptides, which constitute an important aspect of host innate immunity (Bals, 2000). However, it is presently unclear as to whether or not additional genetic elements are involved in establishing resistance to PMB in P. gingivalis.

In this study, we developed a genetic screen to further explore the genetic basis of PMB resistance by $P$. gingivalis. We employed a previously developed transposon-mediated mutagenesis system that utilizes Tn4400' to generate a library of random mutants in $P$. gingivalis ATCC 33277 (Chen et al., 2000; Tang et al., 2000). The resulting mutants (approximately 2,700 independent clones) were subjected to a simple screen to identify PMB-sensitive mutants. The identification and characterization of a single PMB-sensitive clone that was obtained by this procedure is described in this report.

\section{Materials and methods}

\section{Bacterial strains and growth conditions}

P. gingivalis (ATCC 33277) was obtained from our stock collection. Bacteria were grown in TYHK medium consisting of $30 \mathrm{mg} \cdot \mathrm{mL}^{-1}$ trypticase soy broth (Becton Dickinson, Sparks, USA), $5 \mathrm{mg} \cdot \mathrm{mL}^{-1}$ yeast extract (Becton Dickinson, Sparks, USA), and $1 \mu \mathrm{g} \cdot \mathrm{mL}^{-1}$ vitamin $\mathrm{K}_{3}$ (menadione) (Sigma-Aldrich, St. Louis, USA). The basal TYHK medium was sterilized by autoclaving, followed by the addition of filter-sterilized hemin (SigmaAldrich, St. Louis, USA) to a final concentration of $1 \mu \mathrm{g} \cdot \mathrm{mL}^{-1}$. Cultures were grown in an anaerobic growth chamber $\left(5 \% \mathrm{H}_{2}, 5 \% \mathrm{CO}_{2}, 90 \% \mathrm{~N}_{2}\right)$ and maintained at $37^{\circ} \mathrm{C}$ on TYHK-agar plates. The $0524 \mathrm{KO}$ strain of $P$. gingivalis (previously designated $1587 \mathrm{KO}$ ), was previously generated by homologous recombination replacement of the PGN_0524 coding region with an erythromycin resistance cassette (Coats et al., 2009). In all experiments, the $0524 \mathrm{KO}$ strain was cultured in TYHK medium supplemented with $5 \mu \mathrm{g} \cdot \mathrm{mL}^{-1}$ erythromycin.
Tn4400'-based mutagenesis of $P$. gingivalis and PMB sensitivity screen

A conjugation method based upon a previously described procedure was used to generate random Tn4400'-mediated insertion mutations in the genome of $P$. gingivalis ATCC 33277 (Chen et al., 2000; Tang et al., 2000). For a typical tri-parental mating, a HB101 strain of E. coli containing pRK231 (plasmid encoding mobilization element) and a DH10B strain of E. coli containing pYT646B (plasmid encoding the Tn4400 transposon) were grown in Luria broth to an $\mathrm{OD}_{600} \sim 0.8$. $P$. gingivalis was grown in $200 \mathrm{~mL}$ TYHK medium to an $\mathrm{OD}_{600} \sim 1.0$, were pelleted and resuspended in $5 \mathrm{~mL}$ of TYHK medium. Subsequently, $1.5 \mathrm{~mL}$ of $P$. gingivalis culture was combined with $0.5 \mathrm{~mL}$ of HB101 (pRK231) and $0.5 \mathrm{~mL}$ of DH10B (pYT646B). The mixture was pelleted, resuspended in $400 \mu \mathrm{L}$ of TYHK, spotted on a $0.45 \mu \mathrm{mol} \cdot \mathrm{L}^{-1} \mathrm{HA}$ nitrocellulose filter (Millipore) that had been placed in the center of a TYHK-agar plate, and incubated aerobically at $37^{\circ} \mathrm{C}$ for $18-20$ hours. After aerobic incubation, the bacterial mixture was re-suspended in $2 \mathrm{~mL}$ of TYHK medium and $100 \mu \mathrm{L}$ aliquots of the suspension were plated on TYHK-agar plates containing $1 \mu \mathrm{g} \cdot \mathrm{mL}^{-1}$ tetracycline (to select for the $\mathrm{Tn} 4400$ ' configuration of Tn4400) and $100 \mu \mathrm{g} \cdot \mathrm{mL}^{-1}$ gentamycin (to counter-select $E$. coli). Following 8-10 days of incubation in an anaerobic chamber at $37^{\circ} \mathrm{C}$, individual colonies were picked and inoculated into $100 \mu \mathrm{L}$ TYHK medium containing $1 \mu \mathrm{g} \cdot \mathrm{mL}^{-1}$ tetracycline and cultured for 2 days anaerobically at $37^{\circ} \mathrm{C}$. To conduct the PMB screen, approximately $2 \mu \mathrm{L}$ of each culture was replica plated on TYHK plates containing either no PMB or $50 \mu \mathrm{g} \cdot \mathrm{mL}^{-1} \mathrm{PMB}$ and scored for $+/$ - growth after two days.

\section{Identification of the PGN_0524 gene coding sequence as the site of TN4400' integration in the chromosome of $P$. gingivalis}

A genomic DNA preparation was prepared from clone 8-1. Polymerase chain reactions (PCR) using clone 8-1 genomic DNA were performed using primers that are specific for the 5'- and 3'flanking regions of PGN_0524 ( SJ163 and SC337 respectively) in combination with primers that are 
specific for the right hand side (' $a$ ') or left hand side ('c') of the Tn4400' insertion element (Chen et al., 2000) to determine the orientation of the Tn4400' transposon within the PGN_0524 locus (Figure 1). Subsequent sequencing analyses of the resulting PCR fragments were performed using the primers that interact with the 5 '-end (primer SJ149) or the 3'-end (primer SJ150) of the coding sequence to determine the site of transposon integration. The sequences of the primers used in this study are indicated as follows:

Primer a: 5'-CAATAATCGACCTCGTAAAAGACT-3'

Primer c: 5'-TAGCAAACTTTATCCATTCAG-3'

Primer SJ149: 5'-ATAGGCCTATGTTTTTGGAATACATT CTTGAAGTAGA-3'

Primer SJ150: 5'-ATTCTAGATCAGAAGAGCAGGAAG ACATAG-3'

Primer SJ163: 5'-ATGAGAACGCCTGTACTACGCC-3'

Primer SC337: 5'-ACAGTAAAGCTTGCGCAAGAGTAT GATCTAC-3,

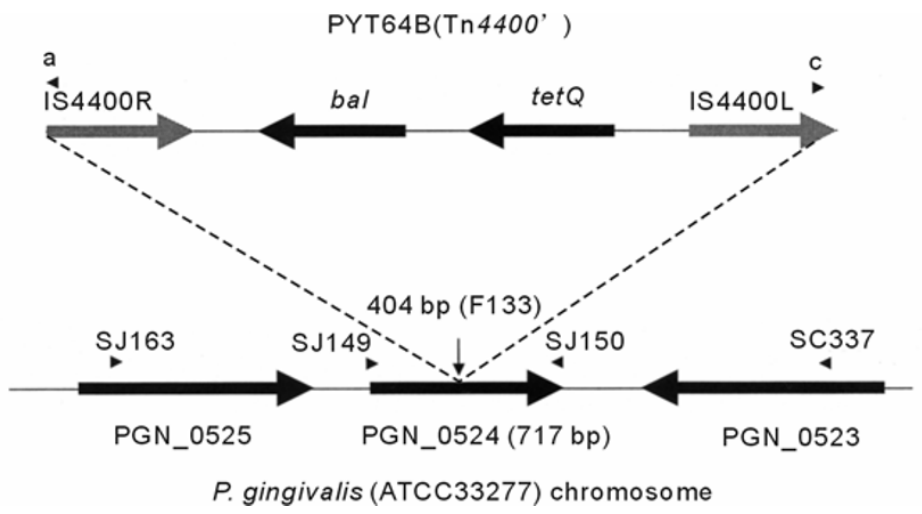

Figure 1 Random mutagenesis of the P. gingivalis ATCC 33277 genome with Tn4400' generates a PMB-sensitive mutant bearing a transposable element integration in the PGN_0524 gene

\begin{abstract}
Schematic representation of the PMB-sensitive clone (8-1) obtained from a screen of 2,700 colonies and the position and orientation of the transposable element, Tn4400', inserted into PGN_0524. Small arrows represent primers used in this study. The orientation of the Tn4400' in the PGN_0524 locus was determined by PCR analyses using primers derived from the upstream (primer SJ163) and downstream (primer SC337) flanking regions of the PGN_0524 gene in combination with primers derived from the left (primer a) and right (primer c) ends of the insertion sequences of Tn4400' (grey shaded arrows) (Chen et al., 2000). Sequencing of the PCR fragments derived from these PCR analyses using primers that interact with the 5'-end of the coding sequence (primer SJ149) and the 3'-end of the coding sequence (primer SJ150) revealed that Tn4400' was integrated at base-pair (bp) position 404 bp of the 717 bp PGN_0524 coding sequence (vertical arrow). This integration event results in a disruption of the protein product at phenylalanine 133 (F133).
\end{abstract}

\section{PMB sensitivity assay}

Overnight cultures of wild-type $P$. gingivalis 33277 and the derivative isogenic mutant strains, 0524KO and 0524-Tn4400', were grown in THYK media containing hemin $\left(1 \mu \mathrm{g} \cdot \mathrm{mL}^{-1}\right)$. Wild-type $P$. gingivalis, 0524KO, and 0524-Tn4400' bacterial starter cultures were subsequently inoculated into $5 \mathrm{~mL}$ TYHK media cultures (initial $\mathrm{OD}_{600}=0.1$ ) containing various doses of $\mathrm{PMB}(0,2.5,5,25,50$, 100 , and $200 \mu \mathrm{g} \cdot \mathrm{mL}^{-1}$ ) and grown in an anaerobic chamber at $37^{\circ} \mathrm{C}$ for 24 hours. Final $\mathrm{OD}_{600}$ was measured for each sample. For each strain, the percent growth was calculated by dividing the $\mathrm{OD}_{600}$ of the cultures containing PMB by the $\mathrm{OD}_{600}$ of the cultures without PMB to determine PMB susceptibility for the respective strains.

\section{LPS and lipid A isolation}

Bacteria were cultured for 48 hours in TYHK medium containing $1 \mu \mathrm{g} \cdot \mathrm{mL}^{-1}$ hemin. LPS was isolated using a modified version of the Trireagent protocol for LPS isolation as previously described (Al-Qutub et al., 2006). To generate lipid A, dried LPS samples were resuspended in $10 \mathrm{mmol} \cdot \mathrm{L}^{-1}$ sodium acetate $[\mathrm{pH} 4.5]$ containing $1 \%$ sodium dodecyl sulfate $(W / V)$. The solution was heated $100^{\circ} \mathrm{C}$ for 1 hour followed by lyophilization overnight. The resulting lipid A pellets were washed once in ice-cold $95 \%$ ethanol containing $0.02 \mathrm{~mol} \cdot \mathrm{L}^{-1} \mathrm{HCl}$, three times in $95 \%$ ethanol, followed by a final extraction with $1,160 \mu \mathrm{L}$ of chloroform-methanol-water $(1: 1: 0.9, \quad V: V: V)$ to remove residual carbohydrate contaminants. The 
chloroform layer containing the lipid A was dried and used for MALDI-TOF MS analysis.

\section{MALDI-TOF analysis}

For MALDI-TOF MS analyses, lipid A samples were dissolved in $10 \mu \mathrm{L}$ of a solution containing the matrix, 5-chloro-2-mercaptobenzothiazole $\left(20 \mathrm{mg} \cdot \mathrm{mL}^{-1}\right)$, dissolved in a mixture of chloroform/methanol 1:1 $(V / V)$. Subsequently, $0.5 \mu \mathrm{L}$ of each sample was analyzed in both positive and negative ion modes on an AutoFlex Analyzer (Bruker Daltonics). Data were acquired with a $50 \mathrm{~Hz}$ repletion rate and up to 3,000 shots were accumulated for each spectrum. Instrument calibration and all other tuning parameters were optimized using HP Calmix (Sigma-Aldrich, St. Louis, USA). Data was acquired and processed using flexAnalysis software (Bruker Daltonics).

\section{Endothelial cell E-selectin expression assay}

Primary human umbilical endothelial cells (passaged 3-5 times) (Clonetics, San Diego, USA) were plated in a 96-well plate format. Endothelial cells were stimulated (in triplicate) for 4 hours at $37^{\circ} \mathrm{C}$ with either isolated LPS or intact cells (as indicated in the figure legend), that had been mixed by vortexing in cell stimulation medium containing $5 \%$ human serum. Endothelial cell E-selectin expression was detected by a previously described ELISA protocol (Reife et al., 2006) and data were plotted using GraphPad Prism software. Data sets were normalized to the unstimulated control and expressed as fold E-selectin expression. Asterisks illustrated above the relevant data points in Figure 4 indicate statistically significant $P$-values $(<0.01)$ that were obtained by unpaired $t$-test analyses.

\section{Results}

Random genomic mutagenesis of $P$. gingivalis with the TN4400' transposon identifies the gene, PGN_0524, as a major determinant for PMB resistance

We performed a Tn4400'-based mutagenesis in $P$. gingivalis (ATCC 33277) according to an established procedure to obtain approximately 2,700 independent colonies containing random genomic insertions of the Tn4400' transposon (Chen et al.,
2000; Tang et al., 2000). The colonies were screened by replica plating on media plates with or without PMB $\left(50 \mu \mathrm{g} \cdot \mathrm{mL}^{-1}\right)$. Only one colony (initially designated clone 8-1) was isolated that was unable to grow when plated on TYHK-agar plates containing PMB $\left(50 \mu \mathrm{g} \cdot \mathrm{mL}^{-1}\right)$. Our studies have shown that $P$. gingivalis bearing a genetically engineered deletion of the entire coding sequence of the gene, PGN_0524, designated 0524KO (previously denoted $1587 \mathrm{KO}$ based upon $P$. gingivalis, strain W83) (Coats et al., 2009) renders this strain highly sensitive to PMB-mediated killing. Therefore, we examined the coding region of the PGN_0524 gene in clone 8-1 to determine if the Tn4400' transposon had inserted into this locus. PCR analyses demonstrated that the coding region for PGN_0524 was indeed modified relative to the genomic sequence present in the wild-type bacterium (data not shown). To determine the orientation of the transposon insertion in the gene, we sequenced the genomic region of this clone using primers derived from the Tn4400 transposable element (Chen et al., 2000) in combination with primers derived from either the 5'- or 3'- flanking regions of the PGN_0524 locus (Figure 1). The results of these analyses demonstrated that the Tn4400' transposon is inserted at nucleotide position 404 of the 717 base-pair coding sequence, which encodes phenylalanine 133 (F133) of the 238 amino acid protein sequence. These data indicate that transposon-mediated disruption of the gene, PGN_0524, in clone 8-1 at amino acid F133 of the protein, produces a strain of $P$. gingivalis (denoted 0524-Tn4400' for the remainder of this study) that exhibits increased sensitivity to PMB $\left(50 \mu \mathrm{g} \cdot \mathrm{mL}^{-1}\right)$. These experiments also clearly demonstrate that $T$ n4400'-based mutagenesis in combination with a rational phenotypic screen is a powerful method to isolate novel genetic determinants of virulence in $P$. gingivalis.

\section{P. gingivalis strains 0524KO and 0524-Tn4400' display similar dosage sensitivities to PMB}

The observation that strain 0524- $\operatorname{Tn} 4400$ ' (clone 8-1) was sensitive to PMB (50 $\left.\mu \mathrm{g} \cdot \mathrm{mL}^{-1}\right)$ and contained an interruption in PGN_0524 strongly suggests that the insertion of Tn4400' in this locus is the underlying basis for the increased sensitivity of 0524-Tn4400' to PMB-dependent killing. Our

- 130 - Int J Oral Sci, 1(3): 126-135, 2009 
previous analyses of the $0524 \mathrm{KO}$ strain, which bears a deletion of the entire PGN_0524 coding sequence, revealed that the $0524 \mathrm{KO}$ mutant is also sensitive to PMB-dependent killing relative to the wild-type strain (Coats et al., 2009). To confirm that both 0524-Tn4400' and 0524KO strains display similar sensitivities to PMB, we compared the relative sensitivities of wild-type $P$. gingivalis, 0524-Tn4400', and 0524KO, to PMB-dependent killing (Figure 2). The results of these experiments demonstrate that both the 0524-Tn4400' and 0524KO strains display identical sensitivities to killing by PMB over a broad dosage range (0$\left.200 \mu \mathrm{g} \cdot \mathrm{mL}^{-1}\right)$ that fails to kill wild-type $P$. gingivalis.

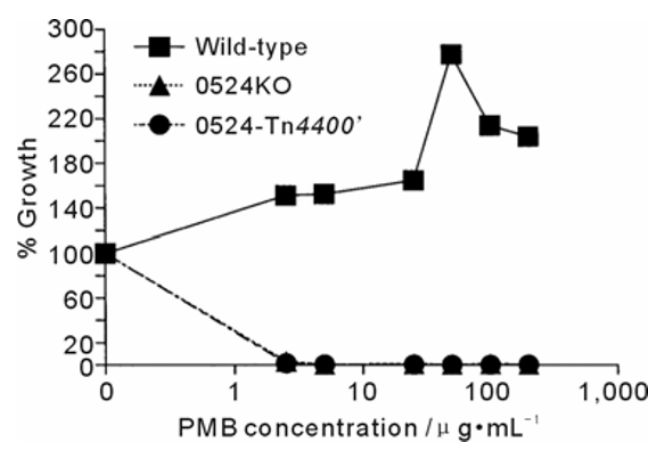

Figure 2 P. gingivalis strains bearing a Tn4400, insertion in PGN_0524 (0524-Tn4400') or a genomic deletion of PGN_0524 (0524KO) display similarly increased dosage-dependent sensitivities to $\mathrm{PMB}$ as compared to wild-type $P$. gingivalis

\section{P. gingivalis 0524-Tn4400' and 0524KO strains exhibit dramatic reductions in the accumulation of non-phosphorylated, tetra-acylated lipid A species relative to wild-type $P$. gingivalis}

We have previously shown that loss of the lipid A 4'-phosphatase, PGN_0524, in the 0524KO mutant results in a drastic reduction in the accumulation of a non-phosphorylated, tetra-acylated lipid A species $(m / z$ 1368) concomitant with the increased accumulation of a mono-phosphorylated, penta-acylated lipid A species ( $m / z$ 1688) (Coats $e t$ al., 2009). In that study, we used MALDI-TOF MS analyses to detect the major lipid A species present in the wild-type strain as compared to the 0524KO strain. Specifically, the use of the positive ion mode MALDI-TOF MS analysis permitted efficient detection of the non-phosphorylated, tetra-acylated lipid A structure $(\mathrm{m} / z$ 1368) that accumulates in wild-type bacteria, but is absent from $0524 \mathrm{KO}$ bacteria. To compare the diagnostic lipid A structures that were derived from wild-type bacteria, 0524KO bacteria, and 0524-Tn4400' bacteria, we performed a similar positive ion mode MALDI-TOF MS analysis (Figure 3). These data demonstrate that wild-type $P$. gingivalis is enriched for the non-phosphorylated, tetra-acylated lipid A ( $\mathrm{m} / \mathrm{z}$ 1368), whereas the $0524-\mathrm{Tn} 4400$ ' and 0524KO strains fail to accumulate this lipid A species, but are enriched for the mono-phosphorylated, penta-acylated lipid A ( $m / z$ 1688) (compare Figures $3 \mathrm{~A}, 3 \mathrm{~B}$, and $3 \mathrm{C}$ ). Therefore, the loss of PGN_0524 function, through either gene deletion (0524KO), or transposable element-mediated gene disruption (0524-Tn4400'), yield strikingly similar lipid A profiles. These data strongly support the hypothesis that PGN_0524 is a critical genetic determinant necessary for the production of the non-phosphorylated, tetra-acylated-acylated lipid A structure that predominates in $P$. gingivalis. These results are also consistent with the idea that it is the loss of lipid A 4'-dephosphorylation, resulting from the disruption of PGN_0524, which renders $P$. gingivalis susceptible to $\mathrm{PMB}$.

\section{Both 0524-Tn4400' and $0524 \mathrm{KO}$ strains activate E-selectin expression in human endothelial cells more potently than wild-type $P$. gingivalis}

Another important characteristic associated with the $0524 \mathrm{KO}$ strain of $P$. gingivalis is that it displays an enhanced ability to stimulate host TLR4 responses in human endothelial cells as compared to wild-type $P$. gingivalis LPS or whole bacteria (Coats et al., 2009). To determine whether or not the 0524-Tn4400' strain displayed similar capabilities, the relative potencies of LPS derived from wild-type $P$. gingivalis, $0524 \mathrm{KO}$, and 0524Tn4400', or the respective bacteria to elicit TLR4depndent E-selectin responses in endothelial cells were compared (Figure 4). Notably, the LPS derived from both $0524 \mathrm{KO}$ and 0524-Tn4400' were significantly more potent activators of E-selectin expression as compared to LPS isolated from wild-type $P$. gingivalis (Figure 4A). Furthermore, preparations of intact $05244 \mathrm{KO}$ and 0524-Tn4400' bacteria displayed a dose-dependent activation of E-selectin expression that was significantly more potent than intact wild-type $P$. 

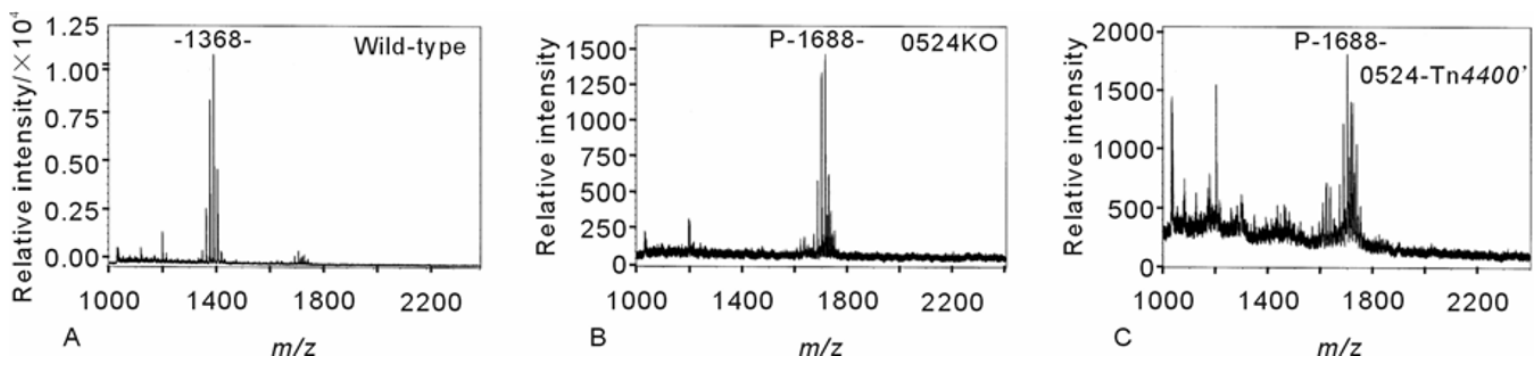

Figure 3 The P. gingivalis mutant strains, 0524-Tn4400' and 0524KO, both fail to generate non-phosphorylated, tetra-acylated lipid A structures relative to wild-type $P$. gingivalis

Positive ion-mode MALDI-TOF MS Lipid A analyses of preparations derived from (A) wild-type, (B) 0524KO, and (C) 0524-Tn4400' strains of $P$. gingivalis, show that both the $0524 \mathrm{KO}$ and the $0524-\mathrm{Tn} 4400^{\prime}$ mutant strains display a pronounced increase in the pentaacylated mono-phosphorylated lipid A (P-1688-), and a marked reduction in the accumulation of the tetra-acylated non-phosphorylated lipid A ion (-1368-) relative to the wild-type strain of $P$. gingivalis.
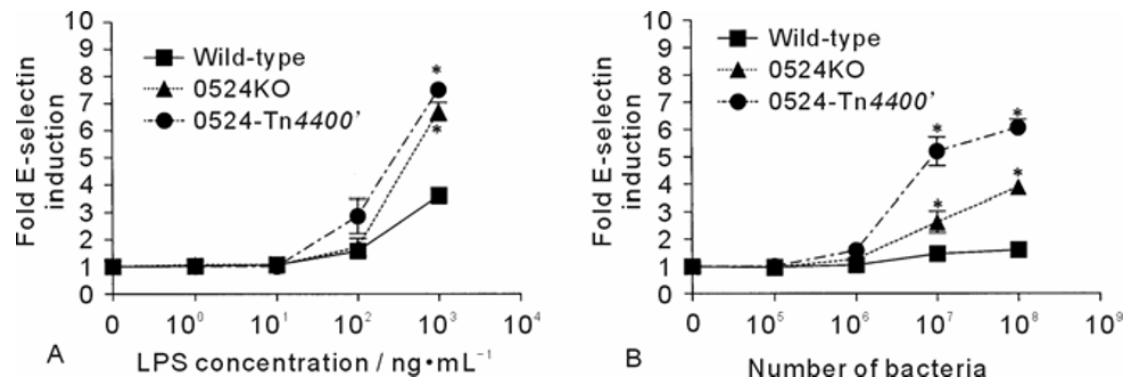

Figure 4 Both $0524-\mathrm{Tn} 4400$ ' and $0524 \mathrm{KO}$ strains of $P$. gingivalis potently activate innate immune responses in human endothelial cells relative to wild-type bacteria

Mutant strains $0524 \mathrm{KO}$ and $0524-\mathrm{Tn} 4400$ ' activate E-selectin expression more potently than wild-type $P$. gingivalis in human endothelial cells. (A): Relative abilities of LPS derived from wild-type, 0524KO, or 0524-Tn4400' P. gingivalis to activate E-selectin expression in human endothelial cells. (B): The relative abilities of intact wild-type, 0524KO, or 0524-Tn4400' bacteria to activate E-selectin expression in human endothelial cells. Standard deviations are indicated by error bars. In both (A) and (B), asterisks indicate statistically significant differences $(P<0.01$; unpaired $t$-test) between mutant and wild-type bacteria.

gingivalis bacteria (Figure 4B). These data demonstrate that the disruption of PGN_0524 in $P$. gingivalis by either gene deletion $(0524 \mathrm{KO})$ or transposon insertion (0524-Tn4400') significantly increase the ability of this bacterium to activate TLR4-dependent host innate immune responses in endothelial cells.

\section{Discussion}

In this study, we took advantage of a transposonmediated random mutagenesis strategy combined with a simple phenotypic screen (PMB sensitivity) to probe the genome of $P$. gingivalis for genetic elements responsible for conferring resistance to cationic antimicrobial peptides. Transposon-mediated mutagenesis in $P$. gingivalis has been successfully used to identify novel genes involved in the regulation of gingipain anchoring and expression as well as fimbriation, suggesting that it is a simple and powerful strategy to identify novel virulence factors in this bacterium (Watanabe-Kato et al., 1998; Simpson et al., 1999; Shoji et al., 2002). The utility of the Tn4400' transposon system for generating random genomic mutations in $P$. gingivalis was previously demonstrated (Chen et al., 2000). In the present study, our identification of a PMB-sensitive mutant derived from a random Tn4400'-generated library gives additional validation for the utility of the Tn4400' system in $P$. gingivalis for identifying novel virulence factors by a simple phenotypic screen.

The main finding of this study is that the gene 
encoding the lipid A 4'-phosphatase, PGN_0524, is the genetic element that confers the pronounced resistance exhibited by $P$. gingivalis towards the cationic antimicrobial peptide, PMB. Multiple genetic strategies used by Gram-negative bacteria to evade the action of PMB have been described including decoration of the lipid A with aminoarabinose or phosphoethanolamine (Gunn et al., 1998; Murray et al., 2007). The requirement for lipid A 4'-phosphatase activity to evade cationic antimicrobial peptides has also been described in the pathogen, Francisella novicida (Wang et al., 2007). The molecular basis for the ability of the lipid A 4'-phosphatase, PGN 0524, to establish cationic antimicrobial peptide resistance in $P$. gingivalis is likely due to removal of the lipid A 4'-phosphate moiety. This modification reduces the negative charge potential of the bacterial outer membrane which is believed to be important for productive interaction with positively charged PMB (Gunn et al., 1998; Wang et al., 2007). However, the removal of the 4'-phosphate also appears to be required for the 3'-O-deacylation of the pentaacylated lipid A to generate non-phosphorylated tetra-acylated lipid A species (Coats et al., 2009). It is presently unclear how either lipid A 3'-Odeacylation or lipid A 1-dephosphorylation influences PMB resistance in $P$. gingivalis. It is interesting to note that lipid A 1-dephosphorylation is important in conferring PMB resistance in the bacterium, Helicobacter pylori (Tran et al., 2006). The accumulation of lipid A lacking phosphates at both the 1- and 4'- positions in P. gingivalis (Rangarajan et al., 2008; Coats et al., 2009) suggests that lipid A 1-phosphate removal might also play a role in the resistance to PMB. Paradoxically, deletion of the lipid A 1-phosphatase, PGN_1713, which contributes to the production of non-phosphorylated lipid A in P. gingivalis, fails to influence the PMB resistance (Coats et al., 2009). Nevertheless, it should be noted that even in the absence of PGN_1713 lipid A 1-phosphatase activity, lipid A 1-dephosphorylation persists suggesting that $P$. gingivalis encodes an additional lipid A 1-phosphatase activity in its genome. Identification of the lipid A 3'-O-deacylase and additional lipid A 1-phosphatase activities should help to resolve the potential roles of these activities in establishing PMB resistance in P. gingivalis.
The mechanism that we have described for PMB resistance suggests that $P$. gingivalis uses lipid A 4'-dephosphorylation by PGN_0524 to evade critical aspects of host innate immunity that are mediated by cationic antimicrobial peptides including cathelicidins and $\beta$-defensins (Bals, 2000). The induction of resistance against human $\beta$-defensins has been described for $P$. gingivalis (Shelburne et al., 2005). In addition, $P$. gingivalis has been reported to resist killing by the cathelicidin, LL-37, as well as $\beta$-defensin 3 which constitute important mechanisms of leukocyte and epithelial cellmediated antimicrobial host (Bals, 2000; Yang et al., 2004; Ji et al., 2007; Reddy et al., 2009). Interestingly, the ability of $P$. gingivalis to evade LL-37-dependent killing closely parallels its ability to resist neutrophil phagocytosis (Ji et al., 2007). Future experiments will be aimed at determining the role of PGN_0524 in evading endogenous host cationic antimicrobial peptide activity. These types of experiments should contribute to elucidating the in vivo role that this phosphatase performs in determining the bacterium's ability to escape host innate immune killing defenses.

Finally, a major implication of the lipid A 4'-phosphatase activity controlling the production of non-phosphorylated lipid A extends beyond the context of $P$. gingivalis to other important Gramnegative pathogens that exhibit phenotypic properties consistent with innate immune evasion. Given the utility of positive ion mode MALDI-TOF MS analysis to efficiently detect non-phosphorylated lipid A in the present study and our recent report (Coats et al., 2009), re-examination of lipid A isolates from other pathogens using this analytical approach should reveal the prevalence of nonphosphorylated lipid A as a potential mechanism to evade and subvert host defenses.

\section{Conclusion}

We used Tn4400'-mediated mutagenesis of the $P$. gingivalis genome to demonstrate that the gene, PGN_0524, which encodes lipid A 4'-phosphatase activity, is responsible for the ability of $P$. gingivalis, to resist high doses of PMB and to evade endothelial cell TLR4 responses. These data strongly suggest that PGN_0524 is the key viru- 
lence factor that determines the ability of the oral pathogen, $P$. gingivalis, to evade host innate immune defenses involving cationic antimicrobial peptide killing and TLR4 sensing.

\section{Acknowledgements}

We appreciate the gift of pYT646B from Dr. Michael H. Malamy. This study was supported by NIH grant (DE 12768) to R. P. D.

\section{References}

Al-Qutub MN, Braham PH, Karimi-Naser LM, Liu X, Genco CA, Darveau RP (2006). Hemin-dependent modulation of the lipid A structure of Porphyromonas gingivalis lipopolysaccharide. Infect Immun, 74(8): 4474-4485.

Bals R (2000). Epithelial antimicrobial peptides in host defense against infection. Respir Res, 1(3): 141-150.

Beutler B (2000). Tlr4: central component of the sole mammalian LPS sensor. Curr Opin Immunol, 12(1): 20-26.

Chen T, Dong H, Tang YP, Dallas MM, Malamy MH, Duncan MJ (2000). Identification and cloning of genes from Porphyromonas gingivalis after mutagenesis with a modified Tn4400 transposon from Bacteroides fragilis. Infect Immun, 68(1): 420-423.

Coats SR, Do CT, Karimi-Naser LM, Braham PH, Darveau RP (2007). Antagonistic lipopolysaccharides block $E$. coli lipopolysaccharide function at human TLR4 via interaction with the human MD-2 lipopolysaccharide binding site. Cell Microbiol, 9(5): 1191-1202.

Coats SR, Jones JW, Do CT, Braham PH, Bainbridge BW, To TT, et al. (2009). Human Toll-like receptor 4 responses to $P$. gingivalis are regulated by lipid A 1and 4'-phosphatase activities. Cell Microbiol, 2009 Jul 13. [Epub ahead of print].

Coats SR, Pham TT, Bainbridge BW, Reife RA, Darveau RP (2005). MD-2 mediates the ability of tetra-acylated and penta-acylated lipopolysaccharides to antagonize Escherichia coli lipopolysaccharide at the TLR4 signaling complex. J Immunol, 175(7): 4490-4498.

Coats SR, Reife RA, Bainbridge BW, Pham TT, Darveau RP (2003). Porphyromonas gingivalis lipopolysaccharide antagonizes Escherichia coli lipopolysaccharide at Toll-like receptor 4 in human endothelial cells. Infect Immun, 71(12): 6799-6807.

Darveau RP, Cunningham MD, Bailey T, Seachord C, Ratcliffe K, Bainbridge B, et al. (1995). Ability of bacteria associated with chronic inflammatory disease to stimulate E-selectin expression and promote neutrophil adhesion. Infect Immun, 63(4): 1311-1317.

Gunn JS, Lim KB, Krueger J, Kim K, Guo L, Hackett M, Miller SI (1998). PmrA-PmrB-regulated genes necessary for 4-aminoarabinose lipid A modification and polymyxin resistance. Mol Microbiol, 27(6): 1171-1182.

Hajishengallis G, Martin M, Schifferle RE, Genco RJ (2002). Counteracting interactions between lipopolysaccharide molecules with differential activation of toll-like receptors. Infect Immun, 70(12): 6658-6664.

Hajishengallis G, Wang M, Liang S, Triantafilou M, Triantafilou K (2008). Pathogen induction of CXCR4/ TLR2 cross-talk impairs host defense function. Proc Natl Acad Sci USA, 105(36): 13532-13537.

Ji S, Hyun J, Park E, Lee BL, Kim KK, Choi Y (2007). Susceptibility of various oral bacteria to antimicrobial peptides and to phagocytosis by neutrophils. J Periodontal Res, 42(5): 410-419.

Kawasaki K, Ernst RK, Miller SI (2004). 3-O-deacylation of lipid A by PagL, a PhoP/PhoQ-regulated deacylase of Salmonella typhimurium, modulates signaling through Toll-like receptor 4. J Biol Chem, 279(19): 20044 20048.

Kovach NL, Yee E, Munford RS, Raetz CR, Harlan JM (1990). Lipid IVA inhibits synthesis and release of tumor necrosis factor induced by lipopolysaccharide in human whole blood ex vivo. J Exp Med, 172(1): 77-84.

Kumada H, Haishima Y, Umemoto T, Tanamoto K (1995). Structural study on the free lipid A isolated from lipopolysaccharide of Porphyromonas gingivalis. $J$ Bacteriol, 177(8): 2098-2106.

Kumada H, Haishima Y, Watanabe K, Hasegawa C, Tsuchiya T, Tanamoto K, Umemoto T (2008). Biological properties of the native and synthetic lipid A of Porphyromonas gingivalis lipopolysaccharide. Oral Microbiol Immunol, 23(1): 60-69.

Mansheim BJ, Onderdonk AB, Kasper DL (1978). Immunochemical and biologic studies of the lipopolysaccharide of Bacteroides melaninogenicus subspecies asaccharolyticus. J Immunol, 120(1): 72-78.

Mata-Haro V, Cekic C, Martin M, Chilton PM, Casella CR, Mitchell TC (2007). The vaccine adjuvant monophosphoryl lipid A as a TRIF-biased agonist of TLR4. Science, 316(5831): 1628-1632.

Munford RS, Varley AW (2006). Sield as signal: lipopolysaccharides and the evolution of immunity to Gram-negative bacteria. PLoS Pathog, 2(6): e67.

Murray SR, Ernst RK, Bermudes D, Miller SI, Low KB (2007). pmrA(Con) confers pmrHFIJKL-dependent EGTA and polymyxin resistance on msbB Salmonella by decorating lipid A with phosphoethanolamine. $J$ Bacteriol, 189(14): 5161-5169.

Nair BC, Mayberry WR, Dziak R, Chen PB, Levine MJ, Hausmann E (1983). Biological effects of a purified 
lipopolysaccharide from Bacteroides gingivalis. $J$ Periodontal Res, 18(1): 40-49.

Naito M, Hirakawa H, Yamashita A, Ohara N, Shoji M, Yukitake H, et al. (2008). Determination of the genome sequence of Porphyromonas gingivalis strain ATCC 33277 and genomic comparison with strain W83 revealed extensive genome rearrangements in $P$. gingivalis. DNA Res, 15(4): 215-225.

Njoroge T, Genco RJ, Sojar HT, Hamada N, Genco CA (1997). A role for fimbriae in Porphyromonas gingivalis invasion of oral epithelial cells. Infect Immun, 65(5): 1980-1984.

Peterson AA, Munford RS (1987). Dephosphorylation of the lipid A moiety of Escherichia coli lipopolysaccharide by mouse macrophages. Infect Immun, 55(4): 974-978.

Poltorak A, He X, Smirnova I, Liu MY, van Huffel C, Du $\mathrm{X}$, et al. (1998). Defective LPS signaling in $\mathrm{C} 3 \mathrm{H} / \mathrm{HeJ}$ and $\mathrm{C} 57 \mathrm{BL} / 10 \mathrm{ScCr}$ mice: mutations in Tlr4 gene. Science, 282(5396): 2085-2088.

Raetz CR, Reynolds CM, Trent MS, Bishop RE (2007). Lipid A modification systems in Gram-negative bacteria. Annu Rev Biochem, 76: 295-329.

Rangarajan M, Aduse-Opoku J, Paramonov N, Hashim A, Bostanci N, Fraser OP, et al. (2008). Identification of a second lipopolysaccharide in Porphyromonas gingivalis W50. J Bacteriol, 190(8): 2920-2932.

Reddy CG, Reddy G.G, Kawasaki H, Oya H, Miller LE, Howard MA 3rd (2009). Decoding movement-related cortical potentials from electrocorticography. Neurosurg Focus, 27(1): E11.

Reife RA, Coats SR, Al-Qutub M, Dixon DM, Braham PA, Billharz RJ, et al. (2006). Porphyromonas gingivalis lipopolysaccharide lipid A heterogeneity: differential activities of tetra- and penta-acylated lipid A structures on E-selectin expression and TLR4 recognition. Cell Microbiol, 8(5): 857-868.

Rietschel ET, Kirikae T, Schade FU, Mamat U, Schmidt G., Loppnow H, et al. (1994). Bacterial endotoxin: molecular relationships of structure to activity and function. FASEB $J, 8(2):$ 217-225.

Shelburne CE, Coulter WA, Olguin D, Lantz MS, Lopatin DE (2005). Induction of \{beta\}-defensin resistance in the oral anaerobe Porphyromonas gingivalis. Antimicrob Agents Chemother, 49(1): 183-187.

Shoji M, Ratnayake DB, Shi Y, Kadowaki T, Yamamoto K, Yoshimura F, et al. (2002). Construction and characteri- zation of a nonpigmented mutant of Porphyromonas gingivalis: cell surface polysaccharide as an anchorage for gingipains. Microbiology, 148(Pt 4): 1183-1191.

Simpson W, Wang CY, Mikolajczyk-Pawlinska J, Potempa J, Travis J, Bond VC, Genco CA (1999). Transposition of the endogenous insertion sequence element IS1126 modulates gingipain expression in Porphyromonas gingivalis. Infect Immun, 67(10): 5012-5020.

Socransky SS, Haffajee AD (1994). Evidence of bacterial etiology: a historical perspective. Periodontol 2000, 5: $7-25$.

Socransky SS, Haffajee AD, Cugini MA, Smith C, Kent RL Jr (1998). Microbial complexes in subgingival plaque. J Clin Periodontol, 25(2): 134-144.

Takii R, Kadowaki T, Baba A, Tsukuba T, Yamamoto K (2005). A functional virulence complex composed of gingipains, adhesins, and lipopolysaccharide shows high affinity to host cells and matrix proteins and escapes recognition by host immune systems. Infect Immun, 73(2): 883-893.

Tang YP, Malamy MH (2000). Isolation of Bacteroides fragilis mutants with in vivo growth defects by using Tn4400', a modified Tn4400 transposition system, and a new screening method. Infect Immun, 68 (1): 415-419.

Tran AX, Whittimore JD, Wyrick PB, McGrath SC, Cotter RJ, Trent MS (2006). The lipid A 1-phosphatase of Helicobacter pylori is required for resistance to the antimicrobial peptide polymyxin. J Bacteriol, 188(12): 4531-4541.

Wang X, Ribeiro AA, Guan Z, Abraham SN, Raetz CR (2007). Attenuated virulence of a Francisella mutant lacking the lipid A 4'-phosphatase. Proc Natl Acad Sci USA, 104(10): 4136-4141.

Watanabe-Kato T, Hayashi JI, Terazawa Y, Hoover CI, Nakayama K, Hibi E, et al. (1998). Isolation and characterization of transposon-induced mutants of Porphyromonas gingivalis deficient in fimbriation. Microb Pathog, 24(1): 25-35.

Yang D, Biragyn A, Hoover DM, Lubkowski J, Oppenheim JJ (2004). Multiple roles of antimicrobial defensins, cathelicidins, and eosinophil-derived neurotoxin in host defense. Annu Rev Immunol, 22: 181-215.

Yoshimura A, Kaneko T, Kato Y, Golenbock DT, Hara Y (2002). Lipopolysaccharides from periodontopathic bacteria Porphyromonas gingivalis and Capnocytophaga ochracea are antagonists for human Toll-like receptor 4. Infect Immun, 70(1): 218-225.

*Corresponding author: Stephen R. Coats

Address: The University of Washington, School of Dentistry, Department of Periodontics, Seattle, Washington 98195, USA

Tel: 0012065435043 Fax:0012066167478Ｅ-mail: scoats@u.washington.edu 